\title{
The simulation analysis of composite parabolic concentrator to improve the performance of thermoelectric devices
}

\author{
Zeming $\mathrm{He}^{1,2}$, Ming Yang ${ }^{1,2}, \mathrm{Yu} \mathrm{Ma}^{1,2}$,Ding Ding ${ }^{3}$, and Hang Zhang ${ }^{1,2, *}$ \\ ${ }^{1}$ Institute of Engineering Thermophysics, Chinese Academy of Sciences, Beijing, China \\ ${ }^{2}$ University of Chinese Academy of Sciences, Beijing 100049, China \\ ${ }^{3}$ Institute of Material Research and Engineering, 2 Fusionopolis way, Innovis \#08-03, Singapore
}

\begin{abstract}
The collection and reuse of thermal radiation energy generated by high-temperature objects has always been the focus of attention and research. Here we designed and fabricated a compound parabolic concentrator (CPC) that can be used for infrared radiation energy collection based on non-imaging optical technology. The energy gathered by CPC has a significant effect on the improvement of the surface temperature of objects. The thermoelectric (TE) generator is a good choice to utilize this thermal energy. This paper analyses and discusses the effects of CPC on the performance of thermoelectric model by simulation. The result has well demonstrated that the TE model with CPC has not only a considerable reduction in structure size and material consumption, but also ensures higher output power and efficiency. In addition, we propose that the array of CPC shall prominently enhance the performance of thermoelectric device.
\end{abstract}

\section{Introduction}

The collection and utilization of infrared radiation energy as a potential energy source has always been valued by researchers in current energy shortage, such as the reuse of industrial waste heat (high temperature exhaust gas generates a large amount of infrared radiation energy) or the reuse of solar radiation energy [1-3]. Nevertheless, the transfer of radiation energy does not require a medium, which means that the use of a large amount of radiation energy is inefficient and not easy to control. Recently, the development of optics has made it possible to control the heat transfer of radiant energy. The design of non-imaging optics such as compound paraboloid concentrator can well gather and capture the radiant energy, and improve the availability of radiant energy [4-6].

As a kind of new energy technology, thermoelectric conversion with thermoelectric materials as the core can directly convert the two different forms of thermal and electric energy by using the temperature differences without any external force [7-9]. Accordingly, the thermoelectric devices has been widely used in aviation, military, industrial waste heat utilization and other fields due to its simple and compact structure, no moving parts, and fast response [10-13]. In this work, the effects of compound parabolic concentrator (CPC) on the performance of thermoelectric devices has been researched and discussed.

We designed and fabricated a composite parabolic concentrate with hollow structure by using non-imaging optical technology to harness thermal radiation from hot objects. The relevant contents of CPC can be found in Ref. [14]. Aiming to reduce the costs, the CPC was fabricated via using the $3 \mathrm{D}$ printing and vacuum deposited metal methods (Fig. 1a). We report on an experimental demonstration of using the CPC to gather the thermal radiation energy which is generated by high temperature heat source. To verify the significant effect of CPC on the temperature, we choose different heat source temperature to observe the temperature changes after using CPC. There is no air flow and no other heat source during the experiment, and the distance between the heat source and the temperature receiver which is a thermocouple is fixed at $10 \mathrm{~cm}$. As shown in Fig. 1 (b), the effects of using CPC on temperature rise are soaring gradually along with the increasing heat source temperature.

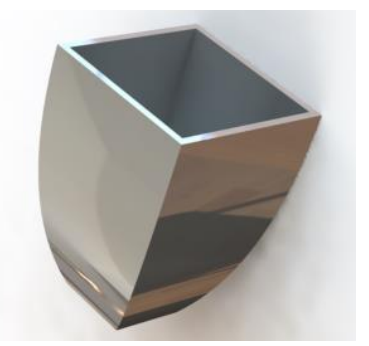

(a)

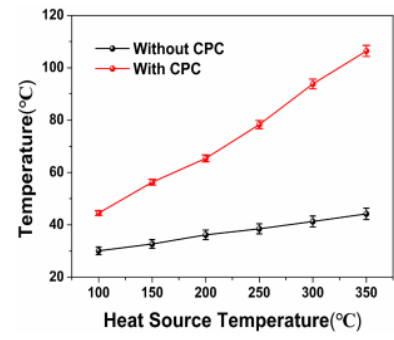

(b)
Fig.1. (a) Schematic of the CPC model (b) The results of the temperature change of receiver with the increase of heat source temperature after using $\mathrm{CPC}$.

Based on the effects of CPC on the temperature rise, here we intend to discuss the effects of CPC on the

Corresponding author: zhanghang@iet.cn 
performance of thermoelectric devices by using the simulation analysis method.

\section{Simulation methods}

\subsection{Geometric model}

The thermoelectric device can be used as the main component of the auxiliary heat and power conversion for waste heat generation [15-17]. In this paper, the TEG1-287 commercialized Bi2Te3 thermoelectric power generation module was numerically simulated. In order to obtain the change in efficiency of the thermoelectric device under different working conditions, numerical analysis is carried out by using the commercial software ANSYS based on finite element analysis. A pair of thermoelectric $p-n$ junction is used as a unit for performance evaluation, as shown in Fig. 2(a). The finite element model of the thermoelectric unit is composed by an upper and a lower ceramic substrate, an electrode conductive layer, a p-type germanium semiconductor, and an n-type germanium semiconductor, wherein the p$\mathrm{n}$ junction has a cross-sectional dimension of 1.5 $\mathrm{mm} \times 1.5 \mathrm{~mm}$ and a height of $1.5 \mathrm{~mm}$. The thickness of the substrate is $0.9 \mathrm{~mm}$, and the thickness of the conductive layer is $0.35 \mathrm{~mm}$ (Fig. 2(b)).

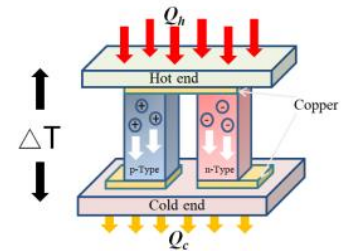

(a)

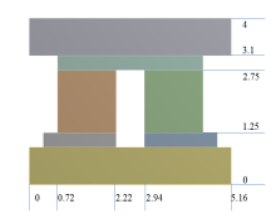

(b)
Fig.2. (a) Schematic of simulation unit of a p-n junction used as a thermoelectric (b) The structure dimensions of the model $(\mathrm{mm})$

The thermal conductivity, electrical conductivity, and Seebeck coefficient of each material are constant, as shown in Table 1, and the governing equations are referred to Ref. [18].

Table 1. Values used for finite element simulations of the TE model in Fig. 2

\begin{tabular}{|c|c|c|c|}
\hline Material & $\begin{array}{c}\text { Thermal } \\
\text { Conductivity } \\
(\mathrm{W} / \mathrm{m} \cdot \mathrm{K})\end{array}$ & $\begin{array}{c}\text { Electrical } \\
\text { Resistivity } \\
(\Omega \cdot \mathrm{m})\end{array}$ & $\begin{array}{c}\text { Seebeck } \\
\text { Coefficient } \\
(\mathrm{V} / \mathrm{K})\end{array}$ \\
\hline $\mathrm{Al}_{2} \mathrm{O}_{3}$ & 22 & - & - \\
\hline $\mathrm{Cu}$ & 385 & $1.75 \times 10^{-8}$ & $6.5 \times 10^{-6}$ \\
\hline $\mathrm{P}-\mathrm{Bi}_{2} \mathrm{Te}_{3}$ & 1.64 & $1.75 \times 10^{-5}$ & $2 \times 10^{-4}$ \\
\hline $\mathrm{N}-\mathrm{Bi}_{2} \mathrm{Te}_{3}$ & 1.47 & $1.35 \times 10^{-5}$ & $-2 \times 10^{-4}$ \\
\hline
\end{tabular}

\subsection{The boundary condition}

The CPC is used to collect and capture the energy emitted in the form of thermal radiation. To place the CPC above the thermoelectric device, the radiation energy is collected by $\mathrm{CPC}$, and the high-intensity radiation energy is directed to the hot end surface of the thermoelectric device. If all losses in this process are ignored, it can be regarded as the heat flow density applied to the hot end of thermoelectric, so that it is regarded as the heat flux specified boundary condition. The first boundary condition is applied to the cold end of thermoelectric model and the temperature is given by:

$$
T(\mathrm{z}=4)=T_{h}=q_{w} \quad T(\mathrm{z}=0)=T_{c}=293 \mathrm{~K}
$$

In the meantime, heat loss along the side surfaces of the model is ignored and implies:

$$
\frac{\partial T}{\partial x}=0, \frac{\partial T}{\partial y}=0
$$

To simplify the calculation process and get more accurate calculation results, several reasonable assumptions are listed as follows: 1) the convective heat transfer on all surfaces is ignored; 2) all materials are isotropic; 3) the contact resistance and thermal resistance are ignored $[19,20]$. In the process of thermoelectric coupling analysis and calculation, the heat conduction, Seebeck effect and Peltier effect of different materials are considered synthetically, and the temperature of load resistance is set as $293 \mathrm{~K}$, which is used to prevent joule heat from accumulating inside of the conductor to avoid the computational divergence. The Thomson effect which has little effects on the calculation results is ignored [21-24].

\subsection{Verification of calculation method}

In order to ensure that the calculation accuracy was not affected by the number of grids, four different grids schemes were tested and the total elements of gird are $\mathrm{i}=$ 8073 , grid ii $=26350$, grid iii $=54580$, grid iv $=119263$, respectively. In general, the output power and conversion efficiency are defined as follows:

$$
\mathrm{P}=\mathrm{U}^{2} / \mathrm{R}_{1}
$$

where $U$ and $R_{1}$ represent load resistance voltage and electric resistance, respectively, and the conversion efficiency can be calculated by:

$$
\eta=\mathrm{P} / \mathrm{Q}_{\mathrm{h}}
$$

where $Q_{h}$ represents heat flow through the hot end of thermoelectric model. The output power and conversion efficiency versus load resistance was calculated using the temperature of the hot end $\left(106{ }^{\circ} \mathrm{C}\right)$ and cold end $\left(20{ }^{\circ} \mathrm{C}\right)$ of thermoelectric unit, with the results illustrated in Fig. 3. The results indicate that almost no difference exists among the curves of four grids, and the maximum deviation is less than $0.5 \%$. Hence, to save computation efforts without loss in accuracy, the grid ii is adopted.

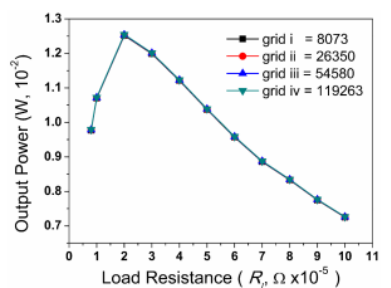

(a)

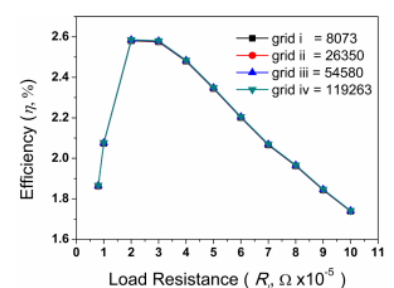

(b)
Fig.3. Comparison between the different grids. (a) Output power and (b) Efficiency 
To further validate the correctness of the model and calculation methods, the thermoelectric device models in the published articles were selected and simulated by methods used in this paper. The relevant parameters of the thermoelectric device are consistent with the data in the Ref. [25]. The current and output power parameters obtained via numerical calculation, for hot end temperatures from $50{ }^{\circ} \mathrm{C}$ up to $120{ }^{\circ} \mathrm{C}$ and cold end temperature set at $30{ }^{\circ} \mathrm{C}$ are presented. As shown in Fig. 4 , the current and output power increases parabolically with the increase of the temperature gradient between the two end of the thermoelectric device, The maximum deviation between the simulation results and the data from Ref. [25] is less than $10 \%$, which is caused by the change of thermoelectric material in physical properties along with temperature and the interface thermal resistance between different materials. However, the simulation results presenting good agreement with the reference data verify the credibility of the modelling procedure.

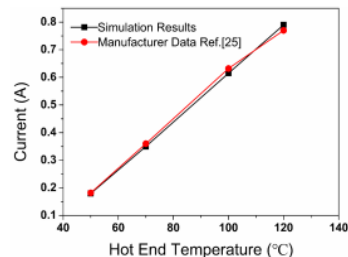

(a)

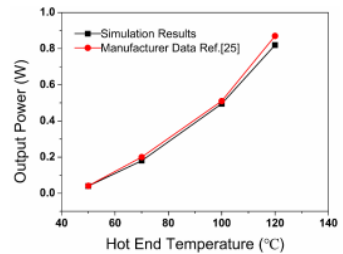

(b)
Fig.4. Comparison between simulation results with reference data. (a) Current and (b) Output power

\section{Results and discussion}

In this paper, the radiation energy collected by $\mathrm{CPC}$ is used to increase the surface temperature of the hot end of the thermoelectric device. Considering the exit diameter of the CPC is $12.5 \mathrm{~mm}$, we assume that the approximate cross section of the $p-n$ junction in the model size selected in this paper is $7.74 \mathrm{~mm}^{2}$. We assume a concentration enhancement of 5.5 times, which is close to the maximum simulated power ratio in Fig. 1. It is evenly distributed on the surface of the thermoelectric device. Assuming that the heat flux density applied to the hot end surface is $10000 \mathrm{~W} / \mathrm{m}^{2}$ (around $400{ }^{\circ} \mathrm{C}$ blackbody with an emitting area of $12 \mathrm{~cm}$ by $12 \mathrm{~cm}$ ), the efficiency of the thermoelectric device changes with load resistance after the energy is accumulated by the CPC such that the hot end experiences a temperature rise of around $62{ }^{\circ} \mathrm{C}$ under maximum load resistance. This is similar to the measured performance of our custom designed $\mathrm{CPC}$ in our experiment in Fig. 1 (d). In the calculation, the temperature distribution and potential distribution of the device module before and after the CPC energy accumulation were obtained, and the influences of the temperature difference on the efficiency of the thermoelectric unit were discussed.

Fig. 5 gives the changes in temperature distribution and potential distribution of a thermoelectric module before and after agglomerating energy by a thermoelectric unit at a maximum efficiency, respectively. It can be clearly seen that under different heat flux conditions, the change trend of the hot end temperature of the thermoelectric unit is basically the same. With CPC, the maximum temperature of the hot end surface increases, and the output potential increases with the increase of the hot end temperature. After the surface of the thermoelectric module receives a high heat flux density, the surface temperature of the hot end rises and the heat is transferred along the thermoelectric arm toward the cold end. During the heat transfer process, the thermoelectric legs generate an output potential due to the Seebeck effect, and as the temperature gradient between the hot end and cold end increases, the output potential also increases.

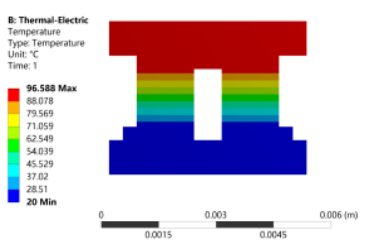

(a)

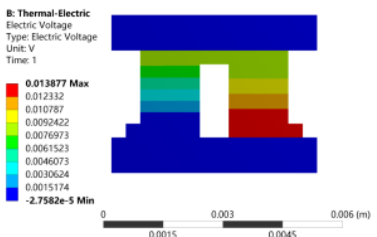

(c)

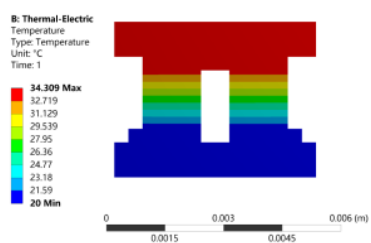

(b)

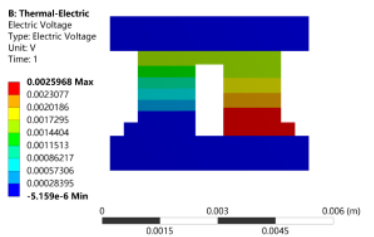

(d)
Fig.5. Simulated temperature (a) with and (b) without $\mathrm{CPC}$ and voltage $(c, d)$ distribution of the thermoelectric unit, $(b, d)$ are the same simulation results without $\mathrm{CPC}$. Thermoelectric unit is connected to load resistance during simulation.

Fig. 6 is a comparison of the output power and efficiency of the thermoelectric unit before and after the energy is accumulated by the CPC. It can be seen that after using the CPC to gather energy, the output power and efficiency of the thermoelectric unit are significantly improved. The maximum efficiency can be achieved at a load of $2 \times 10^{-5} \mathrm{ohm}$. In this case, the output is up to 28 times higher than that without the $\mathrm{CPC}$, and the efficiency is increased by a factor of five. Because the energy concentration results in the rising of the surface temperature of the hot end of the thermoelectric unit, the increase in the temperature difference between the hot end and cold end of thermoelectric unit causes the Seebeck effect of the thermoelectric material to be enhanced, leading to the further increasing of the output power and efficiency. Non-imaging optical concentrating technology has a significant effect on the efficiency of thermoelectric devices.

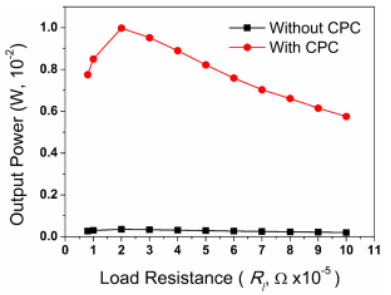

(a)

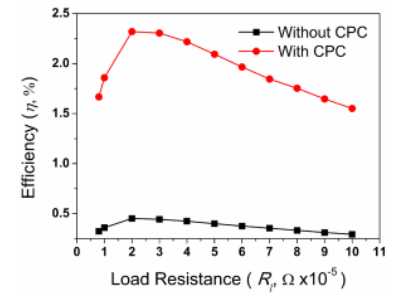

(b)
Fig.6. Simulated output power (a) and efficiency (b) of the thermoelectric unit with and without CPC for different values of load resistance. 
It is difficult to cover the whole surface of the thermoelectric device with the exit area of the concentrated energy of a single CPC, so we can use multiple CPC to form an array, and correspond each $\mathrm{CPC}$ to one or more $\mathrm{p}-\mathrm{n}$ junctions of the thermoelectric device. Meanwhile, the number of $p-n$ junction is optimized, which can increase the output power of the thermoelectric device. The consumption of $p-n$ junction material can be reduced to increase efficiency and reduce weight. Two kinds of models were selected for analysis and comparison to verify the effect of CPC on the output power and efficiency improvement of thermoelectric devices. As shown in Fig. 7, the Model 1 and Model 2 consist of six and two of $p-n$ junctions, respectively. It is assumed that the energy accumulated by CPC can only cover the area corresponding to two pairs of $p-n$ junctions, so as to analysis the influence of $\mathrm{CPC}$ on the thermoelectric performance of the Model 1 without reducing the number of $\mathrm{p}-\mathrm{n}$ junctions. The number of $\mathrm{p}-\mathrm{n}$ junctions in Model 1 is simplified to Model 2, to analysis the effect of CPC under the condition of reducing the number of $p-n$ junctions.

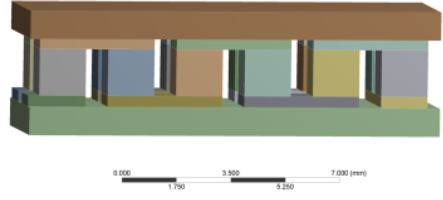

(a)

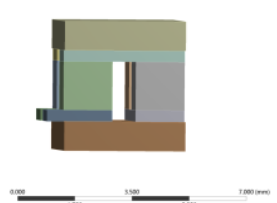

(b)
Fig.7. Two models for analysis and comparison. (a) The Model 1 (b) The Model 2

Fig. 8 is a comparison of temperature and voltage of Model 1 with and without CPC. The radiation energy is concentrated on the surface of two pairs of $p-n$ junctions by $\mathrm{CPC}$, which leads to the local temperature rise of the surface of thermoelectric model and the heat transfer to the surrounding. The average temperature of the hot end of Model 1 is increased to compare with the situation without CPC. As shown in Fig. 8 (a) and (b), the area of high-temperature in Model 1 with $\mathrm{CPC}$ is significantly larger than that without $\mathrm{CPC}$, which increases the temperature difference between hot end and cold end of thermoelectric model. According to the characteristics of thermoelectric materials, the high temperature difference is beneficial to increasing the output power and efficiency of thermoelectric device, and similar conclusion can be drawn from the voltage distribution in Fig. 8 (c) and (d). Therefore, an array is formed by multiple CPC and the accumulated energy is covered to each p-n junction, the output power and efficiency of the thermoelectric device will be prominently improved.

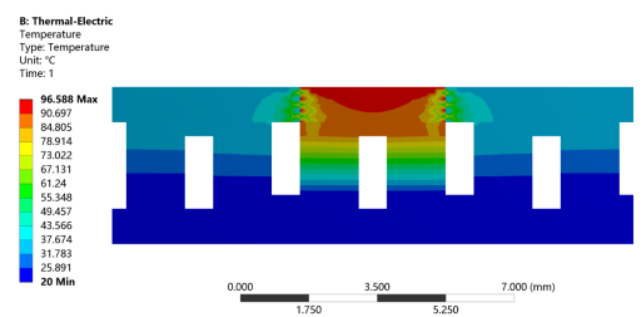

(a)

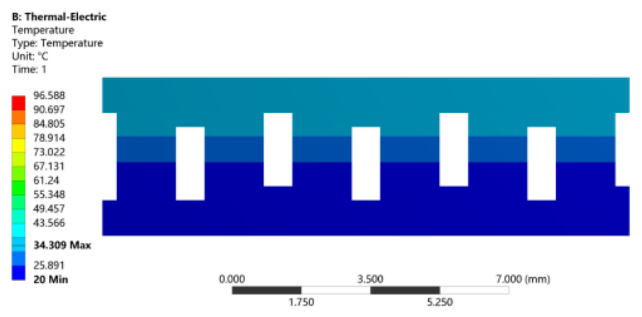

(b)

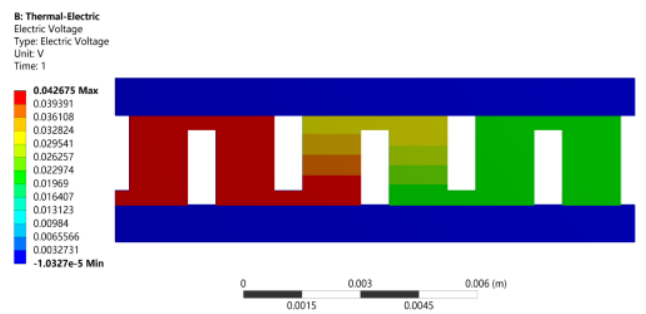

(c)

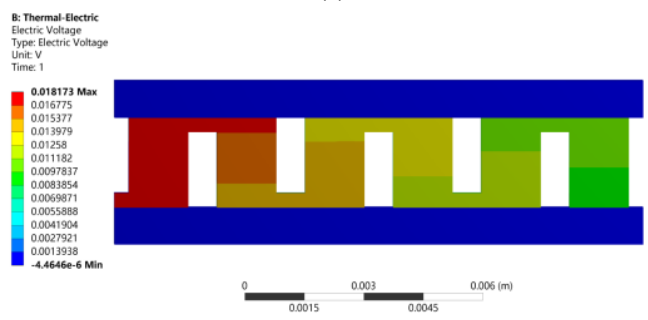

(d)

Fig.8. Simulated temperature $(a, b)$ and voltage $(c, d)$ distribution of Model 1 with and without CPC, (a) and (c) are the same simulation results with CPC. Thermoelectric model is connected to load resistance during simulation.

Compared with Model 1, the number of $p-n$ junctions in Model 2 is reduced by $66 \%$. The output power of Model 1 is larger than that of Model 2 under the same temperature difference between the hot end and cold end of thermoelectric model, which is caused by the difference in the number of $\mathrm{p}-\mathrm{n}$ junctions that implicated in Fig. 9 (a). With CPC, the output power and efficiency of Model 1 can be increased by 5.5 times and 2.5 times, respectively.

In general, when the load resistance value is close to the internal resistance value, the maximum output power and conversion efficiency of the thermoelectric device can be obtained. The different number of $p-n$ junctions leads to the different internal resistance values of the thermoelectric model, which is one of the reasons for the inconsistency in the efficiency trends of Model 1 and Model 2, as illustrated in Fig. 9 (b). Compared with the situation without $\mathrm{CPC}$, the output power and efficiency of Model 1 with CPC are maximum increased by 5.80 times and 2.48 times, respectively. Comparatively, that of Model 2 with CPC are maximum increased by 28 times and 5 times. It is obvious that the best output power and efficiency can be obtained at a load of $4 \times 10^{-5}$ ohm in Model 2 with CPC. Compared to the Model 1 with CPC, the output power and efficiency can be maximum increased to 5 times and 6.8 times, respectively, which illustrate the use of CPC can reduce the consumption of $p-n$ junction materials while ensuring the high output power, showing highlight effect in optimizing the number of $\mathrm{p}-\mathrm{n}$ junctions of thermoelectric device. 


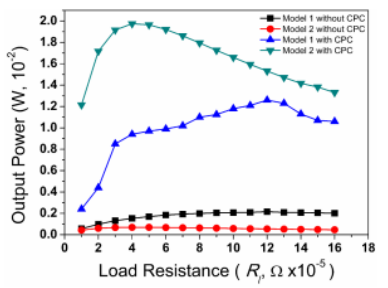

(a)

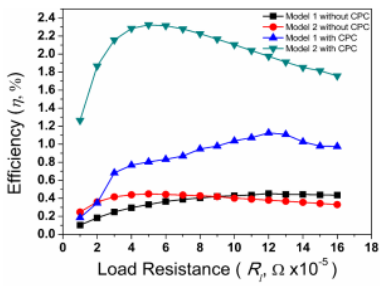

(b)
Fig.9. Simulated output power (a) and efficiency (b) of the Model 1 and Model 2 with and without CPC for different values of load resistance.

\section{Conclusions}

Aiming to collect the radiation energy generated by hot objects or waste heat, a kind of compound paraboloid concentrator was designed by non-imaging optical technology and further demonstrated by simulation analysis in this paper. The energy accumulated by the CPC can significant change the surface temperature of the objects. The performance of a thermoelectric device with CPC was simulated and the results demonstrated significant enhancement in both output power and efficiency. The output power and efficiency of the Model 2 with CPC are maximum increased by 5 times and 6.8 times than that of Model 1 with CPC, respectively. In addition, we proposed that the use of CPC can reduce the number of $p-n$ junctions in thermoelectric model. As a rule of thumb, the cost of semiconductor materials for making p-n junctions is much higher than that of CPC. The combination of CPC and thermoelectric device can not only reduce the cost, weight and materials consumption, but also improve efficiency and output power. This brings a method for the optimization of internal structure of thermoelectric device and waste heat recovery. Compared with the system without CPC, although the use of CPC leads to a more complex system operation, it can effectively save space while maintain or improve the output power for large-scale demand, which is another advantage.

\section{Acknowledgments}

This work is supported by the Basic Science Center Program for Ordered Energy Conversion of the National Nature Science Foundation of China (No. 51888103 and No. 51606192) and the CAS Pioneer Hundred Talents Program.

\section{References}

1. Z. Y. Xu, R. Z. Wang, Chun Yang, Energy, 176, 1037-1043 (2019)

2. Anton Firth, Bo Zhang, Aidong Yang, Applied Energy, 232, 1314-1334 (2019)
3. Y Gao, Z Wang, D Ding, W Li, Y Ma, Y Hao, H Zhang, ES Energy \& Environment, 5, 1-7 (2019)

4. A. RABL, Sol. Energy, 18, 93-111 (1975)

5. T. T. Zhu, Y. H. Diao, Y. H. Zhao and F. F. Li, Appl. Therm. Eng., 98, 1201-1212 (2016)

6. M. Tian, Y. H. Su, H. F. Zheng, G. Pei, G. Q. Li, S. Riffat, Renewable and Sustainable Energy Reviews, 82, 1272-1296 (2018)

7. L. Chen, J. Gong, F. Sun, C. Wu, Int. J. Therm. Sci., 41, 95-99 (2002)

8. E. F. Thacher, B. T. Helenbrook, M. A. Karri, C. J. Richter, Eng., 221, 95-107 (2007)

9. M. F. Remeli, A. Date, B. Orr, L. C. Ding, B. Singh, N. D. N. Affandi, et al., J. Power. Sources, 252, 264269 (2014)

10. Y.X. Zhen, M. Yang, H. Zhang, G. S. Fu, J. L. Wang, S. F. Wang, R. N. Wang, Science Bulletin, 62, 1530-1537 (2017)

11. H Xi, L Luo, G Fraisse, Renew Sustain Energy Rev, 11, 923-936 (2007)

12. Y. X. Zhen, M. Yang, R. N. Wang, Frontiers of Physics, 14, 23603 (2019)

13. Y. X. Zhen, M. Yang, H. Zhang, G. S. Fu, J. L. Wang, S. F. Wang, R. N. Wang, Science Bulletin, 62, 1530-1537 (2017)

14. Z. M. He, M. X. Foo, D. Yong, T. Ma, Y. Hao, H. Zhang, D. Ding, ES Energy \& Environment, 6, 7884 (2019)

15. Y Tian, Z Miao, X. N. Meng, W. S Yan, M. Y. Z, Energy Procedia, 158, 1559-1564 (2019)

16. Yoshikazu Shinohara, Materials Today: Proceedings, 4, 12333-12342 (2017)

17. Y Du, J Xu, B Paul, P Eklund, Applied Materials Today, 12, 366-388 (2018)

18. E. E. Antonova, D. C. Looman, ICT 2005. 24th International Conference on Thermoelectrics, 215218 (2005)

19. Shifa Fan, Yuanwen Gao, Energy, 150, 38-48 (2018)

20. Y. Ge, Z. C. Liu, H. N. Sun, W. Liu, Energy, 147, 1060-1069 (2018)

21. M. J. Huang, R. H. Yen, A. B. Wang, International Journal of Heat and Mass Transfer, 48, 413-418 (2005)

22. M. J. Liao, Z. He, C. P. Jiang, X. A. J, W. W. F, F. S. Qi, Applied Thermal Engineering, 133, 493-500 (2018)

23. M. Chen, L. A. Rosendahl, T. Condra, International Journal of Heat and Mass Transfer, 54, 345-355 (2011)

24. S.C. Kaushik, S. Manikandan, Energy Conversion and Management, 103, 200-207 (2015)

25. D. N. Kossyvakis, C. G. Vossou, C. G. Provatidis, E.V. Hristoforou, Renewable Energy, 74, 1-10 (2015) 